\title{
Prevalencia y Características de Retinopatía Diabética en la Fundación DONUM, Cuenca, Julio 2016 - Febrero 2017
}

\section{Prevalence and Characteristics of Diabetic Retinopathy in the DONUM Foundation, Cuenca, July 2016 - February 2017}

\author{
Arcos, Marisa* y Meneses Maldonado, Tatiana A. \\ Universidad Católica de Cuenca \\ marcos@ucacue.edu.ec
}

\begin{abstract}
Resumen
El objetivo fundamental es determinar la prevalencia y características de Retinopatía Diabética en la Fundación DONUM de la ciudad de Cuenca en el período Julio 2016 - Febrero de 2017. Se realizó un estudio transversal en 162 pacientes diabéticos que acudieron a la consulta externa de la fundación DONUM de la ciudad de Cuenca durante julio de 2016 a febrero 2017. Se caracterizó a la población de acuerdo a edad, sexo, residencia, valores de glucemia promedio, así como también se estableció las características de retinopatía de los mismos. Los datos obtenidos se presentaron mediante gráficos y tablas y se analizaron mediante estadística descriptiva con la aplicación de razón de prevalencia (RP) y se consideró estadísticamente significativo valores inferiores a p $<0,05$ con un intervalo de confianza (IC) al $95 \%$ y fueron procesados en el programa SPSS 22. En los 162 pacientes la prevalencia de retinopatía diabética (RD) es de 38,3\%, con predominio del sexo femenino, Son mayores a 65 años $(48,4 \%)$, se consideran mestizos el 88,7\%), residencia en zonas rurales $(54,8 \%)$.Los valores de glicemia promedio están entre 100 y $140 \mathrm{mg} / \mathrm{dl}$ el $(58,1 \%)$ y el 83,9\% tienen diabetes mellitus tipo 2. En el 64,5\% se observó RD no proliferativa El $8.1 \%$ RD no proliferativa moderada El $11.3 \%$ presenta RD no proliferativa grave. El 9,7\% presenta RD proliferativa de bajo riesgo y solo el 3,2\% presenta RD proliferativa de alto riesgo; y el 3,2 \% edema macular. La Prevalencia de Retinopatía Diabética en la fundación DONUM es alta, los más afectados son las personas de mayor edad y que padecen diabetes mellitus tipo 2 .
\end{abstract}

Palabras clave: Diabetes mellitus, Retinopatía diabética, Oftalmologia, Glucemia, Retina, Prevalencia.

\begin{abstract}
Objective: To determine the Prevalence and Characteristics of Diabetic Retinopathy in the Foundation DONUM of the city of Cuenca in the period July 2016 - February 2017. Materials and Methods: a cross-sectional study was carried out on 162 diabetic patients who attended the external consultation of the DONUM foundation of the city of Cuenca during July 2016 to February 2017. The population was characterized according to age, sex, residence, Values of glycemia, as well as establishing the characteristics of retinopathy of the same. The data obtained were presented by means of graphs and tables and analyzed by means of descriptive statistics with the application of prevalence ratio (RP), and values below $p<0.05$ were considered statistically significant with a 95\% confidence interval (CI) and Were processed in the SPSS program 22. Results: The prevalence of diabetic retinopathy $(R D)$ was $38.3 \%$ in the 162 patients, with a predominance of females. They were older than 65 years (48.4\%), mestizos (88.7\%), The mean values of glycemia are between 100 and $140 \mathrm{mg} /$ $\mathrm{dl}(58.1 \%)$ and $83.9 \%$ have diabetes mellitus type 2. In $64.5 \%$ Non-proliferative $R D 8.1 \%$ moderate non-proliferative $R D$ $11.3 \%$ have severe non-proliferative RD $9.7 \%$ have low-risk proliferative RD and only $3.2 \%$ have high-risk proliferative RD. And 3.2\% macular edema. Conclusions: The Prevalence of Diabetic Retinopathy in the DONUM Foundation is high, the most affected are the elderly and suffering from type 2 diabetes mellitus.
\end{abstract}

Key words: Diabetes mellitus, Diabetic retinopathy, Ophthalmology, Glycemia, Retina, Prevalence.

\section{Introducción}

La Retinopatía Diabética (RD) es la primera causa de ceguera irreversible en personas de edad productiva (16 a 64 años) en países en vías de desarrollo, por esto, es urgente desarrollar programas para la detección temprana y tratamiento precoz de esta enfermedad. ${ }^{1}$

La Organización Mundial de la Salud (OMS) estima que la retinopatía diabética produce casi $5 \%$ de los 37 millones de ciegos del mundo. La ceguera por RD es prevenible en un $80 \%$ de los casos con una detección y tratamiento temprano asociado a un manejo general. El $10 \%$ de los pacientes con diabetes tienen una limitación visual severa y $2 \%$ de ellos llega a la ceguera. ${ }^{23}$

Afecta a ambos ojos y principalmente se determina por pérdidas de visión y más aún cuando la enfermedad ya no es reversible. ${ }^{23}$ Después de 20 años, el $90 \%$ de los Diabéticos 
tipo 1 y el $60 \%$ del tipo 2 tendrán alguna forma de RD y de ellas el $5 \%$ requerirá de tratamiento. ${ }^{4}$

Los factores de riesgo son: el tiempo de evolución de la Diabetes, cifras de tensión arterial, glicemias y hemoglobina glicosilada (HbA1c), presencia de microalbuminuria y nefropatía diabética, mal control de la propia retinopatía obesidad, niveles elevados de lípidos en sangre, ateroesclerosis, tipo de Diabetes Mellitus y tratamiento con insulina, factores oculares, entre otros. ${ }^{5}$

De acuerdo con las Guías de la Asociación Latinoamericana de Diabetes del año 2013, se establece que para la prevención de la (RD), es necesario: "El óptimo control glicémico (HbA1c de menor a $7 \%$ ), de la presión arterial (menor o igual a $130 / 80 \mathrm{mmHg}$ y de los lípidos en suero $(\mathrm{Col} \mathrm{LDL}<100 \mathrm{mg} / \mathrm{dl}, \mathrm{HDL}>40 \mathrm{mg} / \mathrm{dl}$ y $\mathrm{TG}<150 \mathrm{mg} / \mathrm{dl}){ }^{3}$

La Asociación Americana de Diabetes (ADA) y Asociación Europea para el Estudio de la Diabetes (EASD) han propuesto algoritmos para enfatizar el tratamiento formulando metas de control en donde se tiene en cuenta la edad, el estado de salud, la duración de la enfermedad y las comorbilidades. $^{3}$

La prevalencia de la diabetes en la población general oscila entre $2 \%$ y $4 \%$ y está aumentando a nivel mundial debido al envejecimiento de la población sobre los 60 años. Según la OMS, 170 millones de personas estaban afectadas por diabetes en el año 2000 y aumentarán a 370 millones para el año 2030, lo cual representa $86 \%$ de incremento. En Latinoamérica, aumentarán de 13.3 millones a 33 millones para el año 2030 (incremento de $14.8 \%)^{4}{ }^{4}$

La Diabetes Mellitus (DM) es una enfermedad que afecta a la población en general. Se da por un trastorno en el metabolismo de los hidratos de carbono, disminución de la secreción o de la efectividad de la insulina lo que ocasiona hiperglucemia, acompañada de síntomas como poliuria, polidipsia, polifagia y pérdida o ganancia de peso. La Hiperglicemia crónica de la DM se asocia con daño, disfunción y falla de varios órganos, especialmente los ojos y vasos sanguíneos. ${ }^{67}$

DIABETES MELLITUS TIPO 1. Conocida como diabetes mellitus insulino dependiente, o diabetes juvenil. Es el resultado de la destrucción de las células beta secretoras de insulina del páncreas, la susceptibilidad genética está marcada por los antígenos de histocompatibilidad clase 2 (DR3 y DR4). ${ }^{8}$

Las complicaciones de la DM tipo 1 y DM tipo 2 son agudas: hipoglucemia, hiperglucemia y cetoacidosis diabética (DMT1) y estado hiperosmolar (DMT2) y crónicas, observarse la presencia de complicaciones microangiopáticas, como la Retinopatía Diabética y la Nefropatía diabética incipiente (NDI) y neuropatía diabética. Las complicaciones macrovasculares afectan a los vasos sanguíneos más grandes como la aorta, la carótida o los vasos sanguíneos de las extremidades.

DIABETES MELLITUS TIPO 2. Es un trastorno que se caracteriza por grados variables de resistencia a la insulina, alteración de la secreción de insulina y un aumento de la producción de glucosa, es una enfermedad poco sintomática. Este tipo de diabetes aumenta con la edad, obesidad e inactividad física y habitualmente se asocia a otras enfermedades de alto riesgo cardiovascular, como la hipertensión y la dislipidemia. La Federación Internacional de Diabetes (IDF por sus siglas en inglés) estimó que en el 2011 la prevalencia de diabetes en Latinoamérica era de $9.2 \%$ entre los adultos de 20 a 79 años, solo Norteamérica $(10.5 \%)$ y el Sur de Asia (10.9\%) tenían tasas mayores 1 . De los 371 millones de adultos que viven con diabetes, 26 millones $(7 \%)$ residen en Latinoamérica.

La RD es un tema central de la oftalmología y un problema mundial de salud pública, dado que es una de las principales causas de ceguera en adultos. Es relevante conocer los factores causales y asociados que propician el desarrollo rápido de $\mathrm{RD}$, ya que es asintomática y muchos pacientes desconocen su padecimiento. La pérdida de la visión en la Retinopatía Diabética (RD) se puede producir por la presencia de vasos desarrollados anormalmente y frágiles. La segunda forma sería la presentación del edema macular que se determina con un examen oftalmológico completo (fondo de ojo). Es considerada la principal causa de ceguera en Norteamérica con $10 \%$ de nuevos casos cada año y la principal causa de pérdida visual no recuperable en los países industrializados.

En la (RD), las alteraciones patológicas más tempranas son el engrosamiento de la membrana basal endotelial capilar y alteración del endotelio retinal, que producen filtración de líquidos y de lípidos, asociado a una isquemia retinal que desencadena neovasos, sangramiento intraocular y un desprendimiento de retina traccional.

La prevalencia de acuerdo a un estudio panamericano establece que el $40.2 \%$ presentaba algún grado de retinopatía y $17 \%$ requería de tratamiento, siendo preocupante que $35 \%$ nunca había sido examinado por un oftalmólogo. Por tanto la problemática radica que no hay estudios respecto al tema de Retinopatía Diabética que va en aumento día a día. Por lo que la población experimenta pequeños trastornos de visión, puede empeorar y amenazar hasta con la pérdida de esta. ${ }^{9}$ En los diabéticos tipo 1, la RD se presenta en $98 \%$ de los pacientes con 15 o más años de evolución; y en un $2 \%$ de los que tienen menos de dos años con la enfermedad. ${ }^{10}$

El tratamiento principal de la retinopatía diabética es la prevención y la corrección de los factores de riesgo desde el punto de vista oftalmológico. Es necesario una exploración completa en los diabéticos tipo 1 y luego una revisión anual, mientras que en diabetes tipo 2 el primer examen debe ser realizado en el momento del diagnóstico, y posteriormente, una revisión anual. ${ }^{11}$

El $25 \%$ de los pacientes con Diabetes Mellitus tipo 1 tienen algún grado de RD a los 5 años del diagnóstico y llega cerca de $100 \%$ a los 20 años de evolución. Solo 15$20 \%$ en los diabéticos tipo $2^{12}$ a los 10 años, entre $35 \%$ a $40 \%$ presentan RD, llegando a un $80 \%$ a los 20 años de evolución de este tipo de diabetes. Aproximadamente $2 \%$ 
de pacientes diabéticos estarán ciegos después de 15 años y $80 \%$ presentarán algún grado de retinopatía diabética.

Un estudio en Estados Unidos encontró que 3,4\% de la población analizada tenían Retinopatía Diabética y $0,75 \%$ tenían Retinopatía Diabética con alto riesgo de pérdida visual (Retinopatía Diabética Proliferativa y Retinopatía Diabética No Proliferativa severa). En el grupo de aquellos que conocían su condición de diabéticos, la cifra de Retinopatía Diabética fue de 40,3\% y Retinopatía Diabética, que amenaza la visión de 8,2\%.

En Guanajuato, México, se hizo un seguimiento en 100 diabéticos tipo 2 en población abierta, durante 12 años. La incidencia y progresión de la (RD), proliferativa fue de $14.3 \%$ y el $32 \%$, con glucemia de $193 \mathrm{mg} / \mathrm{dL}$ y de la hemoglobina glucosilada $11 \%$.

En octubre del 2010, en Querétaro, México se realizó el segundo taller de retinopatía con una población abierta, de 1840 pacientes de los cuales 13 eran diabéticos tipo 2; 309 pacientes tenían RD (17\%); y un (91\%) tenían Retinopatía Diabética, en fase no proliferativa, el 9\% RD en fase proliferativa, y edema macular en un $(11 \%)$.

"The Wisconsin Epidemiology Study of Diabetic Retinopathy" es un estudio epidemiológico con un seguimiento a 4, 10 y 14 años publicado en el 2008, que ha permitido conocer la historia natural de la RD y su relación con diferentes factores sistémicos. Sus principales hallazgos son: evidenciar que la RD severa es muy rara en los cinco primeros años de diagnóstico, que el riesgo de progresión a una forma severa aumenta con la duración de la enfermedad y los pacientes con cifras alta de HbA1c o con microalbuminuria tienen un riesgo tres veces mayor de presentar RD a corto plazo.

En Estados Unidos personas con diabetes de 40 años o mayores, tienen $28 \%$ y $40 \%$ retinopatía diabética, el 6,5\% de los individuos tienen Degeneración Macular Asociada a la Edad (DMAE) y $73 \%$ de las personas con retinopatía diabética y $84 \%$ de las personas con DMAE no son conscientes de que tienen esta enfermedad. La retinopatía diabética y la Degeneración Macular Asociada a la Edad (DMAE) son las dos enfermedades oculares más comunes en los Estados Unidos, y ambas condiciones pueden progresar hasta causar discapacidad visual severa.

En Chile, $30 \%$ de los pacientes diabéticos evaluados presentaban una Retinopatía Diabética, y de estos casos, 5 a $10 \%$ requirió de tratamiento con fotocoagulación por riesgo de pérdida visual. ${ }^{2}$

La actualización de la Guía clínica de Retinopatía Diabética para Latinoamérica publicada en el 2016 por la Asociación Panamericana de Oftalmología se muestra que en América Latina, hasta un $40 \%$ de los pacientes diabéticos tiene algún grado de RD. ${ }^{13}$

Un artículo cubano sobre factores de riesgo asociados con la aparición de la retinopatía diabética confirma una prevalencia mayor de RD en las personas con más de 60 años de edad. ${ }^{14}$
En el año 2011 en Cuba se realizó un estudio de tipo caso-control en 153 diabéticos atendidos entre julio y diciembre del año 2008 que confirmó que la hipertensión arterial es un factor de riesgo asociado al desarrollo de la retinopatía diabética. La Revista Cubana de Oftalmología expone que la miopía es efecto protector que evita el desarrollo de la RDP. ${ }^{15}$

Una guía mexicana publicada en el año 2013 plantea que la terapia intensiva en diabéticos que incluye insulina reduce las complicaciones micro y macrovasculares. En el United Kingdom Prospective Diabetes Study, el tratamiento intensivo que incluyó la insulina redujo $12 \%$ las complicaciones microvasculares y 17 a $21 \%$ la retinopatía. ${ }^{16}$

Una investigación realizada en el Hospital Vicente Corral Moscoso de la ciudad de Cuenca en el año 2010 mostró que el $12 \%$ de pacientes diabéticos presentaba retinopatía diabética. En el año 2013 en la ciudad de Quito se publicó un estudio donde se estudiaron los pacientes con diabetes mellitus tipo 2 atendidos en consulta externa de Endocrinología del Hospital General $\mathrm{N}^{\circ} 1$ de las Fuerzas Armadas durante el período 2010 a 2011. Los casos fueron aquellos pacientes con retinopatía diabética diagnosticada durante el control oftalmológico y por cada caso se seleccionaron hasta cinco controles no emparejados, se identificaron 62 casos prevalentes de retinopatía diabética (17.1\%). 29 la pérdida de visión se debe principalmente a edema macular, junto a la falta de perfusión capilar, hemorragia vítrea y desprendimiento de retina. Tiene múltiples fases por lo que se ha determinado que un control adecuado puede ayudar a mejorar el diagnóstico y la progresión de la misma, evitando así la pérdida de la visión.

\subsection{Factores de riesgo}

Diabetes. La Diabetes tipo 1 es más frecuente en niños, jóvenes y adultos antes de los 30 años, en tanto que la Diabetes tipo 2 aparece con mayor frecuencia a partir de los 30-40 años. Muchos investigadores manifiestan que el tratamiento con IGF-I ligado a complejos proteicos pueden mostrar mejorías en el control metabólico en conjunto con la insulina, y que en un futuro podrá ser utilizado fundamentalmente para prevenir las complicaciones de la DM. ${ }^{1718}$

Tiempo de evolución. Es el factor de riesgo más importante en el desarrollo de la Retinopatía Diabética ya sea por la duración de la enfermedad, frecuencia y características clínicas. En los pacientes con Diabetes Mellitus tipo 1 la retinopatía aparece casi siempre después de los 10 años de evolución y a partir de los 15 años la prevalencia de Retinopatía Diabética, es similar en ambos tipos. Un estudio realizado a pacientes con DM tipo 1 tratados con insulina de forma intensiva evidenció un empeoramiento transitorio de la Retinopatía Diabética, en los pacientes que ya la padecían y la aparición de esta en $10 \%$ de los casos. ${ }^{1718}$

Cifras de tensión arterial. En estos pacientes el control de la HTA es tan importante como el control metabólico 
para prevenir el desarrollo y progresión de la Retinopatía Diabética. ${ }^{1718}$

Factores oculares. La aparición de otras enfermedades oculares en pacientes con diabetes mellitus disminuye la prevalencia de la $\mathrm{RD}$, dentro de estas enfermedades se encuentran: glaucoma crónico de ángulo abierto, atrofia del nervio óptico, retinosis pigmentaria, oclusión de la arteria central de la retina, miopía superior a 5 dioptrías, atrofia corioretiniana extensa. ${ }^{1718}$

Moss y otros manifiestan que el mecanismo de acción probablemente se produzca por aumento de los niveles séricos de colesterol, HDL, disminución de la agregabilidad plaquetaria y disminución de los niveles séricos de fibrinógeno. ${ }^{1718}$

\subsection{Clasificación de la retinopatía diabética}

\section{- Sin retinopatía diabética.}

1) Al fondo de ojo sin alteraciones aparente. Y la conducta Optimizar control metabólico: glicemia, HTA, colesterol. Control cada año.

\section{- Retinopatía diabética no proliferativa (RDNP)}
1) Leve (ligera)
2) Moderada
3) Severa
4) Muy severa

- Retinopatía diabética proliferativa (RDP)

1) Sin características de alto riesgo (SINCAR)

2) Con características de alto riesgo (CONCAR)

3) Avanzadas (estadios finales)

Retinopatía no proliferativa leve (Riesgo $<0.5 \%$ RDP). En esta fase más inicial se producen microaneurismas, que son pequeñas áreas de inflamación en forma de globo en los diminutos vasos sanguíneos de la retina. ${ }^{1920}$ Conducta a seguir Optimizar control metabólico: glicemia, HTA, colesterol, control en 1 año.

Retinopatía no proliferativa moderada. (5-20\% progresa a RDP), a medida que la enfermedad progresa, los vasos sanguíneos que alimentan la retina se ocluyen. ${ }^{1920}$ Control en 6 meses con igual control metabólico.

Retinopatía no proliferativa severa. En esta etapa los sanguíneos se bloquean, haciendo que varias partes de la retina dejen de recibir sangre. Entonces estas áreas de la retina envían señales al cuerpo para que haga crecer nuevos vasos sanguíneos. ${ }^{19} \mathrm{Al}$ fondo de ojo se observan: Hemorragias retinales en los 4 cuadrantes, rosarios venosos en 2 cuadrantes e IRMA en 1 cuadrante. La conducta a seguir: Regla 4 x 2 x 1 Derivación para panfotocoagulación Oftalmólogo capacitado

Retinopatía proliferativa. Las señales enviadas por la retina para su alimentación desencadenan el crecimiento de nuevos vasos sanguíneos. Estos son anormales y frágiles. ${ }^{19}$ RD proliferativa sin signos de alto riesgo

Presencia de neovasos en 1-4 cuadrantes y menos de 1/3 papila conducta a seguir: Derivación para panfotocoagulación con Oftalmólogo capacitado
RD proliferativa con signos de alto riesgo Presencia de neovasos en más $1 / 3$ papila, hemorragia preretinal o vítrea conducta a seguir: Derivación para panfotocoagulación y/o vitrectomía con Oftalmólogo especialista.

\section{Materiales y Métodos}

Se trata de un estudio de tipo descriptivo cuantitativo observacional, de corte transversal, la investigación se realizó en la Fundación DONUM en Cuenca durante el período de julio 2016 - febrero 2017. Para el cálculo de la muestra como se desconoce el universo, se tomó en cuenta una investigación realizada en el Hospital Vicente Corral Moscoso de la ciudad de Cuenca en el año 2010, donde se encontró que el $12 \%$ de pacientes diabéticos presentaba retinopatía diabética. Este sería el valor de frecuencia esperada del factor a estudiar, con un porcentaje de error del $5 \%$ y un nivel de confianza de $95 \%$, resultando una muestra de 162 participantes. Se utilizó la entrevista estructurada para obtener la información, la cual se procesó por medio del software estadístico SPSS V22 mediante frecuencia absoluta y porcentajes. Se realizó un examen oftalmológico con oftalmoscopia indirecta que consiste en la observación de fondo de ojo mediante un foco luminoso potente e induciendo una fuerte miopía y la colocación de una lente muy convergente de forma que la retina refleja la luz y la lente proyecta una imagen real e invertida del fondo de ojo situada entre la lente y el observador.

Las variables incluidas en este estudio fueron: Edad, lugar de residencia, instrucción, ocupación, factores asociados (HTA; IMC) enfermedades oculares (miopía, glaucoma, catarata, pterigion, astigmatismo sin afectación ocular) tipo de diabetes mellitus, tratamiento de enfermedad de base, controles metabólicos, valores de glicemia promedio, tipo de RD, tiempo de evolución de la enfermedad, y controles de la RD, tratamiento de RD.

\section{Resultados}

El número total de participantes fueron 162 pacientes, de los cuales el $38.3 \%$ presentan RD (tabla 1), los grupos etarios más representados fueron los $>65$ años $(48.4 \%)$ (tabla 2) con predominio de raza mestiza de un $(88.7 \%)$ de sexo femenino. La mayoría vive en zonas rurales $(54,8 \%)$.

Tabla 1. Distribución de 162 pacientes diabéticos según diagnóstico de retinopatía diabética, Cuenca- 2017

\begin{tabular}{|l|l|l|l|}
\hline \multicolumn{2}{|c|}{ Retinopatía Diabética } & Frecuencia(n) & Porcentaje( \%) \\
\hline \multirow{2}{*}{$\mathrm{Si}$} & 62 & 38.3 \\
\cline { 2 - 4 } & No & 100 & 61.7 \\
\cline { 2 - 4 } & Total & 162 & 100.0 \\
\hline
\end{tabular}

Fuente: Formularios de recolección de datos Autora: Tatiana Meneses

En cuanto a los factores asociados, la mayoría de pacientes presentó hipertensión arterial con el $(58,1 \%)$; la miopía 
Tabla 2. Distribución de 62 pacientes con retinopatía diabética según la edad, Cuenca- 2017

\begin{tabular}{|c|c|c|}
\hline Edad (años) & Frecuencia(n) & Porcentaje( $\%)$ \\
\hline $25-34$ & 1 & 1.6 \\
\hline $35-44$ & 8 & 12.9 \\
\hline $45-54$ & 10 & 16.1 \\
\hline $55-64$ & 13 & 21.0 \\
\hline$\geq 65$ & 30 & 48.4 \\
\hline Total & 62 & 100 \\
\hline
\end{tabular}

Fuente: Formularios de recolección de datos Autora: Tatiana Meneses

Tabla 3. Distribución de 62 pacientes con retinopatía diabética según la presencia de enfermedades oculares, Cuenca- 2017

\begin{tabular}{|l|l|r|r|}
\hline \multicolumn{2}{|c|}{ Enfermedades Oculares } & Frecuencia (n) & Porcentaje (\%) \\
\hline \multirow{5}{*}{} & Pterigión & 7 & 11.3 \\
\cline { 2 - 4 } & Glaucoma & 2 & 3.2 \\
\cline { 2 - 4 } Miopía & 12 & 19.4 \\
\hline Catarata & 11 & 17.7 \\
\hline Astigmatismo & 1 & 1.6 \\
\hline Otras & 2 & 3.2 \\
\hline Ninguna & 27 & 43.5 \\
\cline { 2 - 4 } & Total & 62 & 100 \\
\hline
\end{tabular}

Fuente: Formularios de recolección de datos Autora: Tatiana Meneses

y la catarata fueron las más frecuentes con el $(19,4 \%)$ y $(17,7 \%)$ respectivamente(tabla 3$)$.

Los valores de glicemia promedio están en su mayoría entre 100 y $140 \mathrm{mg} / \mathrm{dl}(58,1 \%)$, y el $(83,9 \%)$ de los pacientes tiene diabetes mellitus tipo 2 (tabla 4 ).

Tabla 4. Distribución de 62 pacientes con retinopatía diabética según los controles metabólicos, los valores de glicemia y el tipo de diabetes, Cuenca- 2017

\begin{tabular}{|c|c|c|}
\hline $\begin{array}{l}\text { Valores de Glicemia } \\
\qquad(\mathrm{mg} / \mathrm{dl})\end{array}$ & Frecuencia (n) & Porcentaje (\%) \\
\hline \begin{tabular}{l|l}
$<100$ \\
\end{tabular} & 1 & 2.3 \\
\hline $100-140$ & 25 & 58.1 \\
\hline$>140$ & 16 & 37.2 \\
\hline No recuerda & 1 & 2.3 \\
\hline Total & 43 & 100 \\
\hline & Frecuencia (n) & Porcentaje (\%) \\
\hline DM Tipo 1 & 10 & 16.1 \\
\hline DM Tipo 2 & 52 & 83.9 \\
\hline Total & 62 & 100 \\
\hline
\end{tabular}

Fuente: Formularios de recolección de datos Autora: Tatiana Meneses

El $(54 \%)$ de los pacientes presenta RD no proliferativa leve y solo el $(3,2 \%)$ presenta RD proliferativa de alto riesgo (tabla 5).

\section{Discusión}

Se analizaron 162 pacientes diabéticos que acudieron a la Fundación DONUM, 62 de ellos presentaron retinopatía diabética con una prevalencia de $38,3 \%$. Una investigación
Tabla 5. Distribución de 62 pacientes con retinopatía diabética según el tipo, Cuenca- 2017

\begin{tabular}{|c|c|c|}
\hline $\begin{array}{c}\text { Tipo de } \\
\text { Retinopatía Diabética }\end{array}$ & Frecuencia (n) & Porcentaje (\%) \\
\hline RD proliferativa leve & 40 & 64.5 \\
\hline RD no proliferativa moderada & 5 & 8.1 \\
\hline RD no proliferativa grave & 7 & 11.3 \\
\hline RD proliferativa de bajo riesgo & 6 & 9.7 \\
\hline RD proliferativa de alto riesgo & 2 & 3.2 \\
\hline Edema macular & 2 & 3.2 \\
\hline Total & 62 & 100 \\
\hline
\end{tabular}

Fuente: Formularios de recolección de datos Autora: Tatiana Meneses

realizada en el Hospital Vicente Corral Moscoso de la ciudad de Cuenca en el año 2010 mostró que el $12 \%$ de pacientes diabéticos presentaba retinopatía diabética. En el año 2013 en la ciudad de Quito se estudiaron los pacientes con diabetes mellitus tipo 2 atendidos en consulta externa de Endocrinología del Hospital General $N^{\circ} 1$ de las Fuerzas Armadas durante el período 2010 a 2011. Los casos fueron aquellos pacientes con retinopatía diabética diagnosticada durante el control oftalmológico, se identificaron 62 casos prevalentes de retinopatía diabética $(17.1 \%)$. Al comparar la prevalencia de Retinopatía Diabética en la región con los resultados obtenidos, se puede observar que la prevalencia de esta patología es alta en la población estudiada. En la Actualización de la Guía clínica de Retinopatía Diabética para Latinoamérica publicada en el 2016 por la Asociación Panamericana de Oftalmología se muestra que en América Latina, hasta un $40 \%$ de los pacientes diabéticos tiene algún grado de RD, cifra muy cercana a la encontrada en nuestro estudio. El grupo de edad de mayor afectación fue $\geq 65$ años; estos pacientes representaron el 48,4\%. Un artículo cubano sobre factores de riesgo asociados con la aparición de la retinopatía diabética confirma una prevalencia mayor de RD en las personas con más de 60 años de edad al compararlas con las menores de 30 años. La edad, entre otros factores, aumenta la frecuencia de alteraciones vasculares (venosas y arteriolares), por lo tanto el riesgo de RD se eleva de modo significativo a medida que es mayor la edad.

En cuanto a autoidentificación étnica predominó la mestiza, de sexo femenino con el $88,7 \% .{ }^{13}$ La mayoría de pacientes vive en zonas rurales $(54,8 \%)$. Estas variables están relacionadas con el tipo de paciente que acude a la Fundación DONUM, el hecho de vivir en zonas rurales y dedicarse a actividades productivas con baja remuneración implica difícil acceso a los servicios de salud. En el área rural no se cuenta con el servicio de médicos oftalmólogos y en la ciudad las consultas con estos especialistas tienen un alto costo. Esta situación puede manifestarse como insuficiente control de la retinopatía diabética y las complicaciones que esto conlleva.

Según los factores asociados a la retinopatía diabética, el $58 \%$ de los pacientes presentaba hipertensión arterial. Desde el punto de vista fisiopatológico, la elevación de 
la presión sanguínea puede producir por sí misma daño vascular, y por ello, puede influir sobre la progresión de la RD. Los resultados de múltiples estudios demuestran que las personas con presión arterial diastólica (PAD) y sistólica (PAS) elevadas, padecen con mayor frecuencia RD, Un estudio publicado en Cuba en el año 2011 confirmó que la hipertensión arterial es un factor de riesgo asociado al desarrollo de la retinopatía diabética. ${ }^{15}$ Además se conoce que la hiperlipidemia, considerando los niveles de colesterol y triglicéridos es otro factor de riesgo relacionado con la RD. ${ }^{20}$

La mayoría de pacientes presentan otras enfermedades oculares, la miopía y la catarata fueron las más frecuentes, con el $19,4 \%$ y $17,7 \%$ respectivamente. En el $43,5 \%$ de los casos no se reportaron patologías de la visión concomitantes. Las patologías oculares pueden actuar como factores agravantes o protectores en la retinopatía diabética. Un artículo publicado en la Revista Cubana de Oftalmología expone que está comprobado que la miopía es efecto protector que evita el desarrollo de la RDP. Es posible que la elongación del ojo, la deformación del polo posterior, el adelgazamiento consecuente y la disminución del flujo sanguíneo produzcan un aumento de la difusión del oxígeno y disminuya la tendencia a la neovascularización. Por otro lado, el glaucoma crónico de ángulo abierto protege de la $\mathrm{RD}$, y puede estar relacionado con la disminución del consumo de oxígeno por la degeneración y apoptosis de las células ganglionares. Se menciona los factores que favorecen el desarrollo de la complicación, como son la atrofia óptica, la retinosis pigmentaria, la oclusión de la arteria central de la retina, las inflamaciones oculares y la cirugía intraocular, específicamente la de cataratas. ${ }^{15}$

Se estudiaron los valores de glicemia promedio en los pacientes con retinopatía diabética, que mostró los siguientes resultados: mantienen valores de $<100 \mathrm{mg} / \mathrm{dl}$ el $2,3 \%$, $100-140 \mathrm{mg} / \mathrm{dl}$ el 58,1\%, >140 mg/dl el 37,2\%. La Sociedad Española de Oftalmología plantea que el mayor control ejercido sobre los pacientes diabéticos permite observar una mejoría en la agudeza visual, observándose también que aumenta el número de pacientes tratados. ${ }^{21}$

Según el tipo de diabetes, el 16,1\% de la población con (RD) presentaba diabetes mellitus tipo 1, mientras que en el $83,9 \%$ era tipo 2. En un estudio realizado en Cuba en el año 2011 sobre retinopatía diabética hubo un total de 35 diabéticos tipo 1 para el 22,9\% y los diabéticos tipo 2 representaron el 77,1\%. ${ }^{22}$ El 93,5\% de los pacientes afectados mantiene tratamiento para la diabetes. La mayor cantidad de personas utiliza dieta más hipoglucemiantes orales, con el 37,9\%. En menor frecuencia reciben insulina más hipoglucemiantes orales $(24,1 \%)$, monoterapia con hipoglucemiantes orales $(24,1 \%)$, monoterapia con insulina $(8,6 \%)$, dieta únicamente $(3,4 \%)$ y dieta más insulina $(1,7 \%)$. Una guía mexicana publicada en el año 2013 plantea que la terapia intensiva en diabéticos que incluye insulina reduce las complicaciones micro y macrovasculares. En el United Kingdom Prospective Diabetes Study, el tratamiento inten- sivo que incluyó insulina redujo $12 \%$ las complicaciones microvasculares y 17 a $21 \%$ la retinopatía. ${ }^{23}$

Se evaluó el tipo de RD en los pacientes encontrándose los siguientes resultados: El 64,5\% de los diagnosticados presenta RD no proliferativa leve, RD no proliferativa moderada el $8,1 \%$, RD no proliferativa grave el $11,3 \%$, RD proliferativa de bajo riesgo el $9,7 \%$, RD proliferativa de alto riesgo el 3,2\% y hubo edema macular en el 3,2\% de los casos. Se observa que la mayoría de personas tienen un bajo progreso de la enfermedad, lo que se traduce a un buen pronóstico en cuanto a limitación visual si se mantiene un buen control. Un estudio publicado en Quito en el año 2016 sobre los factores de riesgo asociados a retinopatía diabética en pacientes con diabetes mellitus tipo 2 expone resultados similares, con una muestra de 196 casos de pacientes con $\mathrm{RD}$, encontraron que 179 pacientes es decir el $91,33 \%$ presentaban RD no proliferativa al momento del diagnóstico de su patología ocular, en tanto que el $8,67 \%$ de los casos se determinó por medio del fondo de ojo que los cambios oculares que tenían eran compatibles con una RD proliferativa. ${ }^{24}$ En España la frecuencia de retinopatía diabética proliferativa está entre el 4 y el $6 \%$ y del edema macular entre el 1,4 y el $7,9 \% .{ }^{25}$ En cuanto al tiempo de diagnóstico de la retinopatía diabética de 5 a 10 años en el 41,9\%. En un estudio cubano del año 2013 sobre los factores de riesgo y severidad de la retinopatía diabética también hubo predominio de retinopatía diabética no proliferativa, en el grupo con retinopatía diabética no proliferativa leve y moderada, el mayor número de pacientes se encontraba con un tiempo de evolución de la enfermedad entre 6 y 10 años. ${ }^{26}$ La periodicidad de los controles oftalmológicos en los pacientes con RD depende del progreso de la patología, un artículo publicado en Ecuador en el año 2016 recomienda que una vez diagnosticada la retinopatía diabética se debe seguir los chequeos de acuerdo a las recomendaciones del oftalmólogo con base al grado de la enfermedad. ${ }^{22}$ Un estudio publicado en el año 2012 en Cuba sobre la caracterización clínica de la retinopatía diabética en diabéticos tipo 2, afirma que más del $90 \%$ de la ceguera por diabetes es evitable, si el paciente accede a un estricto tratamiento y seguimiento oftalmológico.

\section{Conclusiones}

Existe alta prevalencia de retinopatía diabética $(38,3 \%)$ en comparación con los estudios de la región. El grupo etario más frecuente fue el de personas con 65 años o más, con el $48,4 \%$, se determinó que en la población estudiada los más afectados son las personas de mayor edad. Predominaron los pacientes mestizos $(88,7 \%)$. En cuanto a los factores asociados a la retinopatía diabética, la mayor cantidad de pacientes presentó hipertensión arterial con el $58,1 \%$. La mayoría de pacientes presentan otras enfermedades oculares, la miopía y la catarata fueron las más destacadas, con el 19,4\% y 17,7 \% respectivamente.

Las guías clínicas recomiendan controles metabólicos periódicos en los pacientes con RD, el 69,4\% de la pobla- 
ción estudiada se realiza estos chequeos de manera habitual. Los valores de glicemia promedio están en su mayoría $(58,1 \%)$ dentro de los parámetros aceptados (100 y 140 $\mathrm{mg} / \mathrm{dl})$ y el $83,9 \%$ de los pacientes tiene diabetes mellitus tipo 2. El 93,5\% de los pacientes mantiene tratamiento para la diabetes, sobresaliendo los que utilizan dieta más hipoglucemiantes orales, con el 37,9\%.

Según la severidad, se demostró un progreso bajo de la enfermedad en la población estudiada, en el 64,5\% de los casos se observó RD no proliferativa leve y solo el 3,2\% presentó RD proliferativa de alto riesgo.

El 35,5\% de las personas con diagnóstico de retinopatía diabética se realizan controles oftalmológicos cada menos de 6 meses y el $25,8 \%$ lo hace cada 6 meses.

\section{Referencias Bibliográficas}

1. Aliseda D, Berástegui L. Retinopatía diabética. Anales del Sistema Sanitario de Navarra. 2008 00;31:23 - 34. Available from: http://scielo.isciii.es/scielo.php?script= sci_arttext\&pid=S1137-66272008000600003\&nrm=iso.

2. Roman SH, Harris MI. Management of diabetes mellitus from a public health perspective. Endocrinology and Metabolism Clinics of North America. 1997;26(3):443474. Available from: http://linkinghub.elsevier.com/ retrieve/pii/S0889852905702607.

3. de Diabetis AL. Diagnóstico, Control y Tratamiento de la Diabetes Mellitus tipo 2 con medicina basada en evidencias. 2013; Available from: http://www.revistaalad. com/pdfs/Guias_ALAD_11_Nov_2013.pdf.

4. von Bischhoffshausen FB, Castro FM. Guía práctica clínica de retinopatía diabética para latinoamérica. Revista Médico Oftalmólogo. 2011;2011. Available from: http://paao.org/images/Downloads/spanish/pdf/ GuiaDeRetinopatiaDiab\%C3\%A9ticaParaLA2010.pdf.

5. Goday A, Serrano-Rios M, Castell C, Lloveras G. Incidence of Type I Diabetes in Southern Europe. Diabetes Care. 1995;18(5):733-733. Available from: http://care. diabetesjournals.org/cgi/doi/10.2337/diacare.18.5.733.

6. Sánchez Salorio M, Sociedad Española de Oftalmología. Manifestaciones oftalmológicas de las enfermedades generales. Madrid: Sociedad Española de Oftalmología; 2001. OCLC: 912498422.

7. of Noncommunicable Disease Surveillance WHOD. Definition, diagnosis and classification of diabetes mellitus and its complications : report of a WHO consultation. Part 1, Diagnosis and classification of diabetes mellitus. Geneva : World Health Organization; 1999. Diabetes. Available from: http://www.who.int/iris/handle/10665/66040.

8. Manuel E Licea Puig, Harbert Fernández Leyva, Eduardo Cabrera Rode, Elvira Maciques Rodríguez. Frecuencia y características clínicas de la retinopatía diabética en un grupo de personas con diabetes mellitus tipo 2 de diagnóstico reciente. 2003;14(2). Available from: http://scielo.sld.cu/scielo.php?script=sci_arttext\&pid= S1561-29532003000200002\&lng=es\&nrm=iso\&tlng=es.
9. Julio César Molina Martín, Yaimara Hernández Silva, Luis Alberto Molina Martín. Factores de riesgos asociados a retinopatía diabética. 2006;19(2). Available from: http://scieloprueba.sld.cu/scielo.php?script=sci_ arttext\&pid=S0864-21762006000200007\&lng=es\&nrm= iso.

10. Hernández Pérez A, Mijail Tirado Martínez O, Rivas Canino MdC, Licea Puig M, Maciquez Rodríguez JE. Factores de riesgo en el desarrollo de la retinopatía diabética. Revista Cubana de Oftalmología. 2011;24(1):86-99. Available from: http://scielo.sld.cu/scielo.php?pid=S0864$21762011000100009 \&$ script=sci_arttext\&tlng=en .

11. Summary of Revisions for the 1998 Clinical Practice Recommendations. Diabetes Care. 1998 Jan;21(Supplement_1):S2-S2. Available from: http://care.diabetesjournals.org/cgi/doi/10.2337/diacare. 21.1.S2.

2. Group ETDRSR, others. Grading diabetic retinopathy from stereoscopic color fundus photographs-an extension of the modified Airlie House classification: ETDRS report number 10. Ophthalmology. 1991;98(5):786806. Available from: http://www.sciencedirect.com/ science/article/pii/S0161642013380129.

13. Juan Verdaguer Terradella, Francisco G Martínez Castro, Fernando barría von-Bischhoffshausen. Actualización de la Guía clínica de Retinopatía Diabética para Latinoamérica; 2016. Available from: https://www.paao.org/images/Downloads/spanish/pdf/ GuiaClinicaRetinopatiaDiabetica2016.pdf.

14. Licea Puig ME, Maciquez Rodríguez E, Cruz Hernández J. Factores de riesgo asociados con la aparición de la retinopatía diabética. Revista Cubana de Endocrinología. 2007 12;18:0 - 0. Available from: http://scielo.sld.cu/scielo.php?script=sci_arttext\&pid= S1561-29532007000300006\&nrm=iso.

15. Hernández Pérez A, Mijail Tirado Martínez O, Rivas Canino MdC, Licea Puig M, Maciquez Rodríguez JE. Factores de riesgo en el desarrollo de la retinopatía diabética. Revista Cubana de Oftalmología. 2011 06;24:86 - 99. Available from: http://scielo.sld.cu/scielo.php?script=sci_ arttext\&pid=S0864-21762011000100009\&nrm=iso.

6. Gil-Velázquez LE, Sil-Acosta MJ, Domínguez-Sánchez ER, Torres-Arreola LdP, Medina-Chávez JH. Guía de práctica clínica. Diagnóstico y tratamiento de la diabetes mellitus tipo 2. Revista Médica del Instituto Mexicano del Seguro Social. 2013;51(1). Available from: http://www. medigraphic.com/pdfs/imss/im-2013/im131o.pdf.

7. Licea Puig ME, Fernández Leyva H, Cabrera Rode E, Maciques Rodríguez E. Frecuencia y características clínicas de la retinopatía diabética en un grupo de personas con diabetes mellitus tipo 2 de diagnóstico reciente. Revista Cubana de Endocrinología. 2003 08;14. Available from: http://scieloprueba.sld.cu/scielo.php?script=sci_ arttext\&pid=S1561-29532003000200002\&nrm=iso.

18. Moss SE, Klein R, Klein BE. Ten-year 
incidence of visual loss in a diabetic population. 27. Plotz CM, Knowlton AI, Ragan C. The natural history of Ophthalmology. 1994;101(6):1061-1070. Available from: http://www.sciencedirect.com/science/article/pii/ S0161642094312176.

19. Preti RC, Motta AAL, Júnior M, Morita C, Nascimento VP, Monteiro MLR, et al. Relationship between diabetic retinopathy severity and the timespan between the endocrinopathy diagnosis and the first ophthalmic examination. 29. Arquivos brasileiros de oftalmologia. 2010;73(3):240 243. Available from: http://www.scielo.br/scielo.php? pid=S0004-27492010000300006\&script=sci_arttext.

20. von Bischhoffshausen FB, Castro FM. Guía práctica clínica de retinopatía diabética para latinoaméri-30. ca. Revista Médico Oftalmólogo. 2011;2011. Available from: http://paao.org/images/Downloads/spanish/pdf/ GuiaDeRetinopatiaDiab\%C3\%A9ticaParaLA2010.pdf.

21. Romero-Aroca P, Fernández-Alart J, Baget-Bernaldiz M, Méndez-Marín I, Salvat-Serra M. Epidemiología de la retinopatía diabética en pacientes tipo II: Cambios 3 observados en una población entre los años 1993 y 2005, tras los nuevos criterios diagnósticos y un mayor control de los pacientes. Archivos de la Sociedad Española de Oftalmología. 2007;82(4):209-218. Available from: http://scielo.isciii.es/scielo.php?pid=S0365- 32 $66912007000400005 \&$ script=sci_arttext\&tlng=pt.

22. Flores H. Retinopatía diabética: tercera causa de ceguera en Ecuador; 2016. Available from: http://revistavive.com/retinopatia-diabetica-terceracausa-de-ceguera-en-ecuador/.

23. Gil-Velázquez LE, Sil-Acosta MJ, Domínguez-Sánchez ER, Torres-Arreola LdP, Medina-Chávez JH. Guía de práctica clínica. Diagnóstico y tratamiento de la diabetes mellitus tipo 2. Revista Médica del Instituto Mexicano del Seguro Social. 2013;51(1). Available from: http://www. medigraphic.com/pdfs/imss/im-2013/im131o.pdf.

24. Flores Mena KS, Jara Tamayo KN. Factores de riesgo asociados a retinopatía diabética en pacientes con diabetes mellitus tipo 2 entre 30 y 60 años de edad, en el hospital San Francisco de Quito durante el período de enero del 2013 a enero del 2015. Pontificia Universidad Católica del Ecuador. Quito;. Available from: http://repositorio.puce.edu.ec/bitstream/handle/ 22000/10456/Tesis\%20Retinopat\%C3\%ADa\%20Diab\% C3\%A9tica\%20\%E2\%94\%82\%20Karen\%20Flores\%20$\% 20$ Kory\%20Jara.pdf? sequence $=1$ \&isAllowed=y.

25. Vila L, Viguera J, Alemán R. Retinopatía diabética y ceguera en España. Epidemiología y prevención. Endocrinología y Nutrición. 2008 Dec;55(10):459475. Available from: http://linkinghub.elsevier.com/ retrieve/pii/S1575092208758431.

26. Castro Cárdenas K, López Dorta N, Rodríguez Rivero D, Suárez Pérez JC, Llerena Rodríguez JA. Factores de riesgo y severidad de la retinopatía diabética. Revista Cubana de Medicina Militar. 2013;42(2):181-190. Available from: http://scielo.sld.cu/scielo.php?script=sci_ arttext\&pid=S0138-65572013000200007. Cushing's syndrome. The American journal of medicine. 1952;13(5):597-614.

28. Yu Y, Chen H, Su SB. Neuroinflammatory responses in diabetic retinopathy. Journal of Neuroinflammation. 2015;12(1). Available from: http://www. jneuroinflammation.com/content/12/1/141.

9. Fong DS, Aiello L, Gardner TW, King GL, Blankenship G, Cavallerano JD, et al. Diabetic Retinopathy. Diabetes Care. 2003;26(Supplement 1):S99-S102. Available from: http://care.diabetesjournals.org/cgi/doi/10.2337/ diacare.26.2007.S99.

. Fouces Gutiérrez Y, Galindo Reymond K, Navarro Scott M, Díaz Valdivia H. Nuestra experiencia en el tratamiento de la retinopatía diabética con fotocoagulación en la Misión Milagro, en Jagüey Grande. MEDISAN. 2007;11(2). Available from: http://www.redalyc.org/html/ 3684/368444987005/.

1. Albuja Arias XP, Páez Iturralde JA, others. Niveles de hemoglobina glicosilada asociados a retinopatia diabética en pacientes del Hospital General de las Fuerzas Armadas de la ciudad de Quito. Periodo 2010-2011. 2013;Available from: http://www.dspace.uce.edu.ec/handle/25000/2121.

. Trelles Guzmán XW, Zumba Pérez AG. Importancia de la educación en la prevención de las complicaciones crónicas de la diabetes en los pacientes de consulta externa y de los talleres de educación diabetológica, en el Hospital Vicente Corral Moscoso, Cuenca 2009-2010 [B.S. thesis]; 2010. Available from: http://dspace.ucuenca.edu.ec/ handle/123456789/3444.

Recibido: 12 de febrero de 2017

Aceptado: 7 de agosto de 2017 